\title{
Diagnóstico de sífilis congênita: comparação entre testes sorológicos na mãe e no recém-nascido
}

\author{
Diagnostic of congenital syphilis: a comparison between serological \\ tests in mother and respective newborn \\ Claudio Barsanti, Fábio Valdetaro, Edna Maria de Albuquerque Diniz
e Regina Célia de Menezes Succi
}

\begin{abstract}
Resumo Para determinarmos as freqüências de sífilis materna e congênita, procedemos ao estudo da resposta aos testes treponêmicos e não treponêmicos de 1.000 parturientes e seus respectivos conceptos. As amostras de sangue venoso da mãe e do recém-nascido e do cordão umbilical foram testadas pelo método de VDRL. Os testes TPHA e ELISA (IgG, IgM) foram utilizados para confirmar os resultados positivos; entre as mães VDRL positivas foi feita a pesquisa de anticorpos anti-HIV. Encontramos 24 (2,4\%) mães VDRL reativas (da população estudada), todas HIV negativas e, entre seus recém-nascidos, 18 (1,8\%) sangue de cordẫo e $19(1,9 \%)$ sangue venoso positivos. Não houve caso de reatividade nos recém-nascidos sem correspondente positividade materna. O teste de VDRL materno pôde, portanto, ser utilizado, isoladamente, na seleção dos casos de sífilis gestacional e congênita, já que não houve maior sensibilidade diagnóstica através da utilização dos testes treponêmicos, que comparados entre si, mostraramse semelhantes.
\end{abstract}

Palavras-chaves: Sífilis Congênita. Sífilis gestacional. Testes treponêmicos. Testes não treponêmicos.

\begin{abstract}
For the purpose of establishing the incidence of maternal and congenital syphilis among pregnant women at delivery and their respective newborns, a study was carried out to determine treponemic and non-treponemic serology in one thousand $(1,000)$ parturient women and their children at Santa Marcelina Hospital - São Paulo, between June 95 and July 96. All blood samples (maternal venous, umbilical cord and newborn venous) were VDRL-tested, treponemic tests (TPHA, ELISA IgG, ELISA IgM) being applied whenever one of the samples from mother or newborn proved positive. Further, an anti-HIV search was run through ELISA among VDRLpositive mothers. Among the 1,000 parturients, 24 (2.4\%) were found to be VDRL-reactive; 18 (1.8\%) newborn children of these 24 mothers presented positive serology in their umbilical cord blood and $19(1.9 \%)$ in venous blood. No positive newborns were found for negative mothers. From the high occurrence of maternal and congenital syphilis in this group of patients, we propose a VDRL maternal test as a way of selecting gestational and congenital syphilis cases, since this test appeared to be sufficiently capable of such diagnoses. Of the treponemic tests, the ELISA test did not enhance diagnostic sensitivity.
\end{abstract}

Key-words: Maternal syphilis. Congenital syphilis. Treponemic tests. Non-treponemic tests.

Casa de Saúde Santa Marcelina, São Paulo, SP.

Suporte financeiro fornecido pela Coordenação de Aperfeiçoamento de Pessoal de Nível Superior - CAPES.

Endereço para correspondência: Dr. Claudio Barsanti: R. Loefgreen 1654/ 93, Vila Clementino, 04040002 São Paulo, SP.

Fax.: 5511 5084-5050.

Email: barsanti@uol.com.br

Recebido para publicação em 21/9/98. 
A sífilis, embora conhecida pela humanidade há vários séculos, continua como um crescente desafio, sendo considerada, em alguns países subdesenvolvidos e em desenvolvimento, como o principal problema de Saúde Pública 310121721. No Brasil, a sífilis congênita passou a ser de notificação compulsória em 1986, porém, devido à ausência de uniformidade no diagnóstico e conduta, bem como, a falhas no mecanismo de notificação, sabemos que a freqüência da doença é bem maior do que a demonstrada pelos números oficiais. O Ministério da Saúde, em 1993, fazendose valer de números encontrados em estudos epidemiológicos, encontrou que 153.462 mulheres deveriam ser positivas ao exame do VDRL e que, deste modo, poderiam gerar 130.443 crianças com sífilis congênita, embora, menos de 400 casos desta doença tenham sido notificados no mesmo período ${ }^{1822}$.

Mesmo em países desenvolvidos, a doença tem experimentado um importante aumento em sua freqüência, principalmente, na última década. Nos Estados Unidos, em 1988, mais casos de sífilis congênita foram reportados ao Centers for Disease Control (CDC), do que em quaisquer dos 15 anos precedentes ${ }^{8}$.
Por outro lado, a associação da sífilis à síndrome de imunodeficiência adquirida (SIDA), pode conduzir a uma evolução agressiva e, por vezes, fulminante, das duas moléstias, trazendo grande preocupação à comunidade médica ${ }^{212} 1822$.

Assim, tendo em vista o grande número de gestantes com sífilis, suas repercussões na morbimortalidade materna e dos respectivos recém-nascidos, sua relação com a SIDA, a dificuldade na realização do diagnóstico da infecção congênita, bem como, a proposta do Ministério da Saúde de erradicação da sífilis congênita, em nosso país, até o ano 2000, nos propusemos estudar uma população de parturientes e de seus recém-nascidos, a fim de verificarmos a prevalência das doenças (sífilis e SIDA gestacionais e sífilis congênita), o perfil sorológico dessa população e a possibilidade de selecionarmos exames laboratoriais, dentre os mais freqüentemente utilizados para o diagnóstico de sífilis (VDRL, TPHA e ELISA) que, além da determinação da infecção congênita, permitissem um diagnóstico mais fácil, rápido e barato, sem perda de sensibilidade e especificidade.

\section{MATERIAL E MÉTODOS}

A seleção de casos foi realizada na enfermaria de pré-parto da Casa de Saúde Santa Marcelina (CSSM), no período compreendido entre junho de 1995 e julho de 1996. O trabalho foi prospectivo, sendo estudados 1.000 pares mãe-recém-nascidos consecutivos e cujos recém-nascidos tinham peso superior a $1.500 \mathrm{~g}$.

Os tubos com material para análise foram encaminhados ao Laboratório de Patologia Clínica da CSSM dentro das 24 horas subseqüentes à coleta, ficando armazenados em geladeira até o encaminhamento. Uma vez por semana, os soros eram avaliados pelo teste VDRL. Os soros maternos positivos ao VDRL, independente de suas titulações, foram encaminhados para pesquisa de teste treponêmico (TPHA), o mesmo acontecendo com as amostras de sangue de seus correspondentes recém-nascidos e cordões umbilicais. Para os soros maternos reativos foi feita, também, a pesquisa de anticorpos anti-HIV pela técnica de ELISA. Todo o material (sangue materno, de cordão e de recém-nascido) com reação positiva foi submetido a testes para detecção de anticorpos anti-Treponema pallidum das classes IgG e IgM, pela técnica de ELISA, realizados no setor de Imunologia do Laboratório REM de São Paulo.
Procedemos, dentre as mães VDRL negativas, a um sorteio aleatório de 170 soros que foram testados pelo método de ELISA IgG antiTreponema pallidum, nesse mesmo laboratório (REM, SP). Por fim, a esses soros VDRL negativos e que apresentaram reação positiva no ELISA, realizamos, no Laboratório de Patologia Clínica da CSSM, o TPHA e, novamente, as técnicas de VDRL e de ELISA IgG, utilizando, nesta última sorologia, um reagente laboratorial distinto da primeira determinação.

Todas os pares mãe-recém-nascido que tiveram ao menos um teste positivo foram convocados pelo Serviço Social para serem orientados e tratados.

Após a alta hospitalar, as famílias foram encaminhadas para o ambulatório da Disciplina de Infectologia Pediátrica da Escola Paulista de Medicina da Universidade Federal de São Paulo a fim de realizarem os seguimentos clínico e laboratorial necessários.

Para a análise dos resultados foram aplicados os seguintes testes:

1. Análise de Variância por Postos de Friedman ${ }^{23}$ para comparar os títulos de anticorpos anticardiolipina das mães e respectivos recém- 
nascidos e cordões umbilicais determinados pelo método de VDRL. Esta análise foi complementada pelo Teste de Comparações Múltiplas ${ }^{15}$.

2. Teste $\mathrm{G}$ de Cochran ${ }^{23}$ para estudar a presença (+) ou ausência (-) de anticorpos anticardiolipina entre os soros das mães e respectivos recémnascidos e cordões umbilicais, determinados pelo método de VDRL.

3. Teste de Mc Nemar ${ }^{23}$ para estudar as discordâncias entre positividade e negatividade na comparação dos binômios mãe-recémnascido e mãe-cordão umbilical.

4. Testes de Sensibilidade e Especificidade ${ }^{11}$ calculados sobre as tabelas do teste de Mc Nemar.

Observação: em todos os testes fixamos em 0,05 ou $5 \%(a \leq 0,05)$ o nível de rejeição da hipótese de nulidade e assinalamos com um asterisco os valores significantes.

\section{RESULTADOS}

A idade materna variou de 20 a 38 anos, com média, mediana e moda de 28 anos. Dentre todas as pacientes (com exceção de uma de que não conseguimos o dado), 17 tinham história de ao menos uma gestação anterior. Analisando as 13 pacientes com mais de três gestações (considerada a gravidez do presente estudo), encontramos que em 12 delas, o diagnóstico e, eventual tratamento, de sífilis gestacional não havia sido realizado. Em sete histórias clínicas tivemos a citação de abortamentos anteriores.

Quando avaliamos, isoladamente, as 12 pacientes que fizeram seguimento pré-natal considerado como adequado, notamos que nove tiveram diagnóstico de sífilis na gestação. Mesmo quando do diagnóstico, verificamos que apenas três receberam tratamento correto.

As sorologias positivas (qualitativas) do VDRL e as respostas positivas entre mães e respectivos recém-nascidos (sangue venoso e de cordão umbilical) foram comparadas (Tabela 1). Encontramos 24 soros maternos, 19 soros de recém-nascidos e 18 soros de cordão reativos. Não existiu, portanto, em nenhuma tríade, qualquer caso em que o soro materno fosse negativo e pelo menos um de seus correspondentes (cordão

Tabela 1 - Resultados sorológicos comparativos (VDRL).

\begin{tabular}{|c|c|c|c|}
\hline Soros maternos & Soros de cordão & Soros de RN & Total \\
\hline+ & + & + & 17 \\
\hline+ & + & - & 1 \\
\hline+ & - & - & 4 \\
\hline+ & - & + & 2 \\
\hline Total & 18 & 19 & \\
\hline
\end{tabular}

e/ ou sangue periférico de recém-nascido) fosse positivo.

Os títulos das reações VDRL positivas são apresentados na Tabela 2. Comparamos os valores encontrados entre cada membro do conjunto mãe-cordão-recém-nascido, em 21 conjuntos de soros, pois, em três casos, não conseguimos o total de material necessário para a realização do teste quantitativo (Tabela 3 ).

Houve discordância entre o VDRL materno e o de cordão em seis pares de soros (Tabela 1). Ao compararamos as sensibilidades do VDRL materno com as do recém-nascido, encontramos uma discordância um pouco menor (cinco entre 24 soros) (Tabelas 1 e 2), porém, ainda, estatisticamente significante ( $p=0,0312$ ou $3,12 \%$; Teste de Mc Nemar).
Nem todos os soros maternos VDRL reativos foram positivos aos métodos treponêmicos (TPHA e ELISA) (Tabela 4). Porém, quando da comparação entre os dois testes treponêmicos, notamos que houve plena concordância de resultados entre ambos (nos 20 soros em que foi possível a comparação). Ainda, dentre os soros reagentes ao ELISA, apenas um se mostrou positivo à pesquisa de anticorpos da classe IgM (era também IgG positivo).

Por fim, entre os 170 soros maternos sorteados entre as 976 amostras VDRL negativas para serem testadas pela técnica de ELISA IgG, apenas quatro apresentaram reação sorológica positiva. As amostras reativas foram novamente testadas pelos métodos de VDRL, TPHA e ELISA IgG (reagente laboratorial diferente do utilizado 
Tabela 2 - Mães e respectivos recém-nascidos e cordões umbilicais, segundo os valores dos títulos do anticorpo anticardiolipina, pelo método de VDRL.

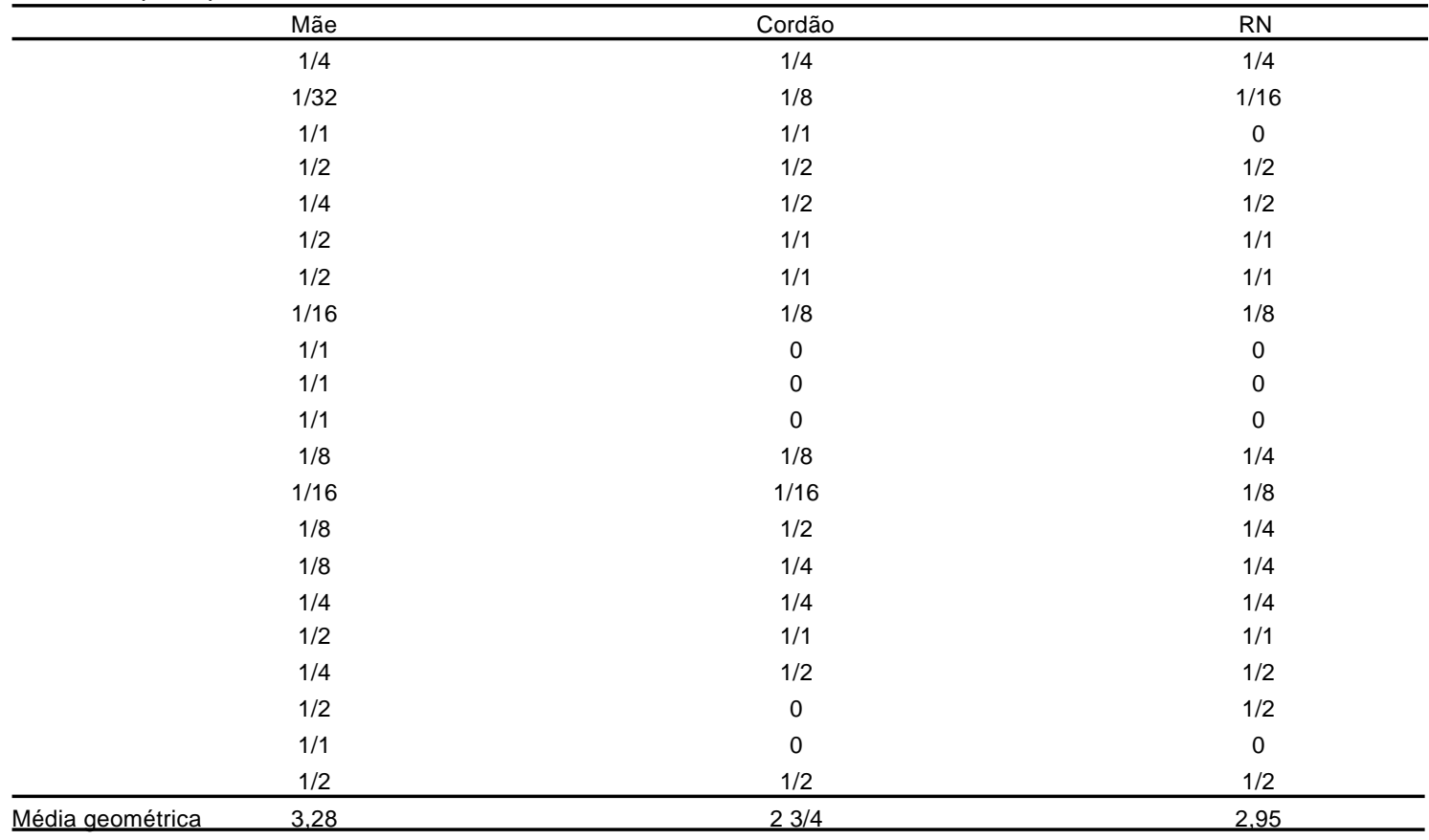

Análise de Variância por Postos de Friedman:

$\mathrm{X}^{2} \mathrm{r}$ calculado $=16,10 \quad * \mathrm{X}^{2} \mathrm{r}$ crítico $=5,99$

na primeira determinação); todas confirmaramse VDRL e ELISA negativas, porém, entre os quatro
Teste de Comparações Múltiplas:

Mãe > cordão e recém-nascido

Tabela 3 - Sensibilidade comparativa entre VDRL de cordão e VDRL materno.

\begin{tabular}{|c|c|c|c|}
\hline & \multicolumn{2}{|r|}{ Mãe } & \multirow[b]{2}{*}{ Total } \\
\hline & + & - & \\
\hline \multicolumn{4}{|c|}{ Cordão } \\
\hline+ & 18 & 0 & 18 \\
\hline- & 6 & 976 & 982 \\
\hline Total & 24 & 976 & 1.000 \\
\hline$\overline{\text { Teste }}$ & & \multicolumn{2}{|c|}{ Teste de Sensibilidade e Especificidade: } \\
\hline \multirow{3}{*}{\multicolumn{2}{|c|}{$p=0,0156$ ou $1,56 \%$}} & Sensibilidade: $\underline{18}=75 \%$ & Especificidade: $\underline{976}=100 \%$ \\
\hline & & 24 & 976 \\
\hline & & Negativo falso $=25 \%$ & Positivo falso $=0 \%$ \\
\hline
\end{tabular}

Tabela 4 - Comparação de resultados sorológicos treponêmicos (TPHA, ELISA) em amostras maternas VDRL +.

\begin{tabular}{cccc}
\hline VDRL & TPHA & ELISA & Total \\
\hline+ & + & + & $\mathbf{1 8}$ \\
+ & + & - & - \\
+ & - & - & $\mathbf{2}$ \\
+ & - & + & - \\
\hline
\end{tabular}

Obs.: 1. Em quatro soros positivos ao TPHA, não foi possível a pesquisa de ELISA, devido à falta de material suficiente para a realização. 2. Um dos soros positivo ao TPHA, foi reagínico aos testes de ELISA, IgM e IgG, concomitantemente. 


\section{DISCUSSÃO}

Apesar do acentuado desenvolvimento da Imunologia e do aparecimento de inúmeros exames específicos para a detecção de infecção pelo Treponema pallidum, o diagnóstico de sífilis (adquirida e congênita) continua fortemente alicerçado no teste de VDRL. Isto se deve à alta sensibilidade (70\% na sífilis primária, $99 \%$ a $100 \%$ na sífilis secundária e $70 \%$ a $80 \%$ na terciária), alta especificidade na sífilis congênita (de até $98 \%)^{4512}$, à facilidade de execução, ao menor custo e à sua indicação, por vários organismos mundiais de saúde, como excelente exame de seleção. Oferece, ainda, a possibilidade de ser utilizado, no seguimento dos casos tratados, na avaliação do processo de cura 52024 . É, ainda, incerta, a possibilidade da gravidez, por si só, causar positividade dos exames não treponêmicos; assim, testes sorológicos positivos em gestantes devem ser atribuídos à infecção sifilítica ${ }^{14} 24$.

O encontro de 24 mães VDRL positivas $(2,4 \%$ das 1.000 gestantes) demonstra a importância da determinação da sorologia de sífilis na população designada. Se considerarmos que de todas as amostras sangüíneas maternas reativas ao teste não treponêmico, apenas duas não apresentaram, simultaneamente, reação aos testes treponêmicos (tanto ao TPHA e ao ELISA IgG e $\lg M$ ), a confirmação do teste não treponêmico ocorreu em $83,3 \%$ dos casos, ou seja, estas 22 pacientes, pelo menos, tinham sífilis no momento do parto e, por conseguinte, seus recém-nascidos deveriam ser tratados de sífilis congênita. Das duas mães restantes, uma havia iniciado o seguimento prénatal no terceiro mês, tendo realizado apenas duas consultas; referia, ainda, tratamento anterior para sífilis, por duas vezes, com terapêutica diferente da preconizada pelo Ministério da Saúde. No segundo caso, a paciente realizou acompanhamento pré-natal adequado a partir da oitava semana e mostrou, por ocasião do retorno a CSSM, resultado de VDRL negativo no quarto mês gestacional.

Em nossa pesquisa constatamos que, partindo da análise dos soros maternos VDRL positivos, todos os demais soros reativos correspondentes, de cordão e de recém-nascido, seriam encontrados; ao contrário, isto é, caso utilizássemos os soros das crianças, ou de seus respectivos cordões, para exames de seleção, algumas mães soro-reativas não seriam diagnosticadas. Assim, quando da necessidade de opção de uma única amostra para o diagnóstico de sífilis gestacional e, por conseguinte, de sífilis congênita, a sorologia materna deve ser a de escolha pela equipe tratante. A possibilidade de resultados negativos falsos, quando da pesquisa de VDRL no cordão umbilical, em se considerando o sangue materno como padrão ouro, foi de $25 \%$ (seis falhas diagnósticas em 24 casos); no caso da utilização do sangue do recém-nascido, esse risco, em relação à amostra materna, foi de $20,8 \%$ (cinco perdas diagnósticas no total de 24). Entretanto, a concordância nestes dois grupos, ainda se considerando o sangue materno como padrão ouro, foi de $100 \%{ }^{11}$.

Os testes treponêmicos são confiáveis na determinação de uma infecção sifilítica, presente ou passada, visto que, eles, normalmente, não se negativam, mesmo após a total cura da infecção, permanecendo positivos por toda a vida do paciente $\mathrm{e}^{202526}$; entretanto, o tratamento precoce pode prevenir a soropositividade e, também, negativar reações já positivas ${ }^{14}$. Assim, não servem para o acompanhamento de cura dos pacientes, mas, para a confirmação de infecção sifilítica em pacientes que já tenham sorologia inespecífica positiva ou, ainda, quando se suspeitar de resultado negativo falso em exame não treponêmico ${ }^{24}$. De realização simples e barata o TPHA não necessita de leitura em microscópio fluorescente, apresentando altas sensibilidade e especificidade ${ }^{1{ }^{13}}{ }^{16}$. Por estas razões, tem sido o exame treponêmico de eleição em vários laboratórios de nosso país ${ }^{12}$. A técnica de ELISA é de fácil realização e de possíveis automatização e computadorização, diminuindo, assim, a chance de erros técnicos humanos ${ }^{25} 26$; de custo não elevado, pode ser utilizada em regiões de baixas condições econômicas, como em países em desenvolvimento, onde a freqüência de sífilis congênita é, normalmente, maior ${ }^{19}$. Por fim, tem a vantagem de determinar anticorpos da classe $\operatorname{lgG}$ e $\operatorname{lgM}$. Foi utilizada, nesta pesquisa, na análise dos soros quando ao menos uma amostra da tríade mãe-cordãorecém-nascido foi VDRL positiva, para que, assim, verificássemos a possibilidade de discordâncias entre os dois métodos treponêmicos. Entretanto, entre todas as amostras que puderam ser testadas pelos dois métodos, houve concordância plena, tanto entre os resultados negativos, como entre os positivos, demonstrando a ausência de resultados positivos falsos e/ou negativos falsos, pelo método de TPHA, quando comparado à 
técnica de ELISA. Assim, embora de fácil realização, esta técnica deve ser indicada, ao nosso entender, como teste treponêmico de segunda escolha, ou, ainda, em casos em que possa existir alguma dúvida diagnóstica pelo método de TPHA.

Neste estudo, na análise das duas amostras em que houve discordância entre os testes não treponêmicos e os testes treponêmicos, ou seja, entre o VDRL e o TPHA e ELISA maternos, constatamos que no primeiro caso a paciente realizou seguimento pré-natal aparentemente adequado, com pesquisa de VDRL negativa no início do acompanhamento. Por ocasião de seu retorno a CSSM, quatro meses após o parto, uma nova pesquisa sorológica, através da reação de VDRL, foi negativa. A outra paciente relatava tratamento para sífilis nas duas gestações anteriores à da pesquisa deste trabalho, porém, com terapêutica inadequada. À sua reavaliação, também, aproximadamente quatro meses após o término da gestação, era VDRL negativa. Entretanto, mesmo nestes dois casos, em um primeiro instante, isto é, durante a internação na maternidade, havia indicação, pelos critérios do $\mathrm{CDC}^{67}$, de tratamento de seus recém-nascidos.

É possível que tenhamos tido alguma criança não portadora da síndrome, mas que, mesmo assim, por preencher os critérios definidos para sífilis congênita ${ }^{6718}$, tenha recebido indicação de tratamento. É fácil compreender, que os riscos de se perder a chance, talvez única, de se tratar crianças doentes, no melhor momento para isso, não se comparam aos riscos que algumas crianças sadias, equivocadamente internadas, poderiam sofrer.

\section{REFERÊNCIAS BIBLIOGRÁFICAS}

1. Alpert G, Plotkin SA. A pratical guide to the diagnosis of congenital infections in the newborn infant. Pediatric Clinics of North America 33:465-479, 1986.

2. Antonio C, Davanzo R. Congenital syphilis in Mocambique: the diagnostic complementary role of laboratory and radiological investigations. Central African Journal of Medicine 35:552-555, 1989.

3. Bam RH, Cronjé HS, Muir A, Griessel DJ, Hoek BB. Syphilis in pregnant patients and their offspring. International Journal of Gynecology and Obstetrics 44:113-118, 1994.

4. Camargo ME. A sífilis avança. Progride o diagnóstico? Revista daAssociação Médica Brasileira 34:19-23, 1988.

5. Camargo ME. Diagnóstico sorológico e métodos de detecção dos agentes etiológicos das infecções congênitas hematogênicas. In: Bittencourt AL(ed) Infecções congênitas transplacentárias, $1^{\underline{a}}$ edição. Revinter. Rio de Janeiro, p.125-150, 1995.

6. Centers for Disease Control - Guidelines for the prevention and control of congenital syphilis. Morbidity and Mortality Weekly Report 37(S1):1-13, 1988.

7. Centers for Disease Control - Congenital syphilis. New York City, 1986-1988. Morbidity and Mortality Weekly Report 38:825-9, 1989.

8. Centers for Disease Control - Congenital syphilis. Morbidity and Mortality Weekly Report 39 (RR13):36-7, 1990.

9. Centers for Disease Control - Syphilis. Morbidity and Mortality Weekly Report 42(RR14):27-46, 1993.

10. Evans HE, Frenkel LD. Congenital syphilis. Clinics in Perinatology 21:149-62, 1994.

11. Gallen RS, Gambino SR. Beyond normality: the predictive value and efficiency of medical diagnoses. John Willey \& Sons, New York, 1975.
12. Guinsburg R. Sífilis congênita. In: Farhat CK (ed) Infectologia pediátrica. $1^{\underline{a}}$ edição, Atheneu, São Paulo, p. 285-95, 1993.

13. Gutman LT. Syphilis. In: Feigin RD (ed) Textbook of pediatric infectious diseases. $3^{\text {rd }}$ edition, WB Saunders, Philadelphia, p. 552-63, 1992.

14. Hart G. Syphilis tests in diagnostic and therapeutic decision making. Annals of Internal Medicine 104:36876, 1986.

15. Hollander M, Wolfe DA. Nonparamedic statistical methods. John Willey \& Sons, New York, p.1-503, 1973.

16. Jaffe HW. The laboratory diagnosis of syphilis. New concepts. Annals of Internal Medicine 83:846-50, 1975.

17. Kilmarx $\mathrm{PH}$, Louis MES. Editorial: The evolving epidemiology of syphilis. American Journal of Public Health 85:1053-4, 1995.

18. Ministério da Saúde. Programa Nacional de Controle de Doenças Sexualmente Transmissíveis e AIDS. Bases técnicas para eliminação da Sífilis congênita, 1993.

19. Pedersen NS, Sheller JP, Ratnam AV, Hira SK. Enzimelinked immunosorbent assays for detection of Immunogloulin $\mathrm{M}$ to nontreponemal and treponemal antigens for the diagnosis of congenital syphilis. Journal of Clinical Microbiology 27:1835-1840, 1989.

20. Pickering LK. Diagnosis and therapy of patients with congenital and primary syphilis. Pediatric Infectious Disease Journal 4:602-605, 1985.

21. Saxoni F, Lapatsanisis P, Pantelakis SN. Congenital syphilis: A description of 18 cases and re-examination of an old but ever-present disease. Clinical Pediatrics 6:68791, 1967. 
22. Secretaria de Saúde do Estado de São Paulo. Divisão de Doenças Sexualmente Transmissíveis/AIDS: relatório sífilis congênita. São Paulo, Centro de Documentação da Secretaria de Saúde, p.1-20, 1993.

23. Siegel S. Estatistica no parametrica. Trillas, Mexico, p.1346, 1975.

24. Starling SP. Syphilis in infants and young children. Pediatric Annals 23:334-340, 1994
25. Stoll BJ, Lee FK, Larsen S, Hale E, Schwartz D, Rice RJ, Ashby R, Holmes R, Nahmias AJ. Clinical and serologic evaluation of neonates for congenital syphilis: a continuing diagnostic dilemma. The Journal of Infectious Diseases 167:1093-1099, 1993.

26. Stoll BJ. Congenital syphilis: evaluation and management of neonates born to mothers with reactive serologic tests for syphilis. Pediatric Infectious Disease Journal 13:845852, 1994. 